\title{
The Accuracy of Crystal Structure Refinement from Electron Diffraction Data using Parallel Beam Illumination
}

\section{J.Jansen}

Nationaal Centrum voor HREM, Technische Universiteit Delft, Rotterdamseweg 137, 2628AL Delft, Nederland.

Crystal structure refine ments using electron diffraction data, obtained by using a parallel electron beam, gives accurate results if a full dynamical calculation is performed to calculate the elastic diffracted intensities from the atomic model[1]. Apart from the dynamical diffra ction it is of utmost importance to take even a slight tilt from the zone axis and the crystal thickness into account during calculation. Our refinement program MSLS, based on the Multi -Slice algorithm, can cope with all

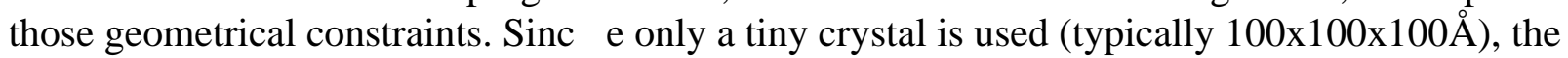
method is extremely powerful in the area of small precipitates, thin layers and multi-phased samples. MSLS has been applied successfully to any type of material whether it being inorganic (i.e. superconductors), metallic or organic compounds. Comparison between the calculated and observed intensities is usually measured in terms of $\mathrm{R}$-values, whose values appear to be of the same order of magnitude as when single crystal X-ray diffraction would have been used.

The definition of the R-values is not unique. MSLS uses R-values of the form:

$$
\mathrm{R}=\Sigma \mathrm{w}_{\mathrm{i}}\left(\mathrm{A}_{\mathrm{obs}, \mathrm{i}}-\mathrm{A}_{\text {calc, },}\right)^{2} / \Sigma \mathrm{w}_{\mathrm{i}} \mathrm{A}_{\mathrm{obs}, \mathrm{i}}{ }^{2}
$$

The symbol A represents here either the (observed and calculated) intensities -in this case we call the $\mathrm{R}$-value $\mathrm{R}_{1}$ - or the square root of the intensities. In the latter case we refer to the $\mathrm{R}$-value as $\mathrm{R}_{2} \cdot \mathrm{w}_{\mathrm{i}}$ represents the weighting scheme. Up to now only unit weights and $1 / \quad \sigma(\mathrm{A})^{2}$ are used to refine structures with MSLS. In the example below R $\quad 1$ and $\mathrm{R}_{2}$ and the two weighting schemes are compared, as a first try to set the $\mathrm{R}$-value criterion in such that the best accuracy o $\mathrm{f}$ the determined parameters is ensured.

Besides the choice of $\mathrm{R}$-value the calculation of the intensities introduces some uncertainty. It is known that the calculation method (i.e. Multi -slice as in MSLS, Bloch waves) does not have a significant effect on the resulting intensities. However, all these methods start from the same atomic scattering factors. Currently, for simulations of electron diffraction patterns and HREM images, the Doyle and Turner table[2] is most frequently used. Several other, more re cent, tables are available in literature [3-5]. In addition some authors modified the atomic scattering factors in order to include an approximation for absorption of the electrons in the crystal[6,7]. Changing from one to the other table gives some change s to the resulting crystal structure obtained by MSLS as shown in the example below.

To illustrate the effect of the different $\mathrm{R}$-values, weighting schemes and atomic scattering factor tables we use diffraction patterns of the [001] -zone of $\mathrm{Ce}_{5} \mathrm{Cu}_{19} \mathrm{P}_{12}$ from nine different areas of which the crystal thickness varied from 80 to $200 \AA$. The crystallographic data are listed in table 1 . For each of the different $\mathrm{R}$-values and scattering factor tables the structural parameters, the crystal thicknesses and the orientation of the zones in respect to the electron beam were refined starting with the structure parameters from the original publication of the structure[1]. An example of the results the $\mathrm{x}$-coordinate of one of the $\mathrm{Ce}$-atoms is plotted in figure 1 as function o $\mathrm{f}$ the $\mathrm{R}$-value/scattering 
factor table. It is clear that inclusion of absorption gives different results compared to the others and that the Doyle and Turner table is even different from the others which do not include absorption. The difference of the coor dinate is of the order of $0.06 \AA$. In the presentation of this paper it will be argued that Doyle and Turner may be the worst choice. It is obvious that the errors estimated from the diagonal elements of the refinement matrix underestimate the errors in the parameters especially in case of $1 / \sigma^{2}$ weights.

[1] J. Jansen et al., Acta Cryst. A54 (1998) 91.

[2] P.A. Doyle et al., Acta Cryst. A24 (1986) 390.

[3] D.T. Cromer et al., Acta Cryst. A24 (1986) 321.

[4] D. Rez et al., Acta Cryst. A50 (1994) 481.

[5] E.J. Kirkland, Advanced Computing in Electron Microscopy, Plenum, New York, 1998.

[6] D.M. Bird et al., Acta Cryst. A46 (1990) 202.

[7] A. Weickenmeier et al., Acta Cryst. A47 (1991) 590.

\begin{tabular}{|l|c|c|c|l|l|c|c|c|}
\hline atom & $\mathrm{x}$ & $\mathrm{y}$ & $\mathrm{z}$ & & atom & $\mathrm{x}$ & $\mathrm{y}$ & $\mathrm{z}$ \\
\hline $\mathrm{Ce} 1$ & $2 / 3$ & $1 / 3$ & 0 & & $\mathrm{Cu} 4$ & $0.6354(8)$ & $0.1165(8)$ & 0.5 \\
\hline $\mathrm{Ce} 2$ & $0.8112(9)$ & 0 & 0.5 & & $\mathrm{Cu} 5$ & $0.4467(6)$ & 0 & 0 \\
\hline $\mathrm{Cu} 1$ & 0 & 0 & 0 & & $\mathrm{P} 1$ & $0.1734(8)$ & 0 & 0 \\
\hline $\mathrm{Cu} 2$ & $0.2868(7)$ & 0 & 0.5 & & $\mathrm{P} 2$ & $0.6457(6)$ & 0 & 0 \\
\hline $\mathrm{Cu} 3$ & $0.3789(6)$ & $0.1776(7)$ & 0 & & $\mathrm{P} 3$ & $0.3171(4)$ & $0.8329(5)$ & 0.5 \\
\hline
\end{tabular}

Table 1 Crystallographic data of $\mathrm{Ce}_{5} \mathrm{Cu}_{19} \mathrm{P}_{12}$. Spacegroup : $\mathrm{P}-62 \mathrm{~m}, \quad a=12.4 \AA, c=4.0 \AA$

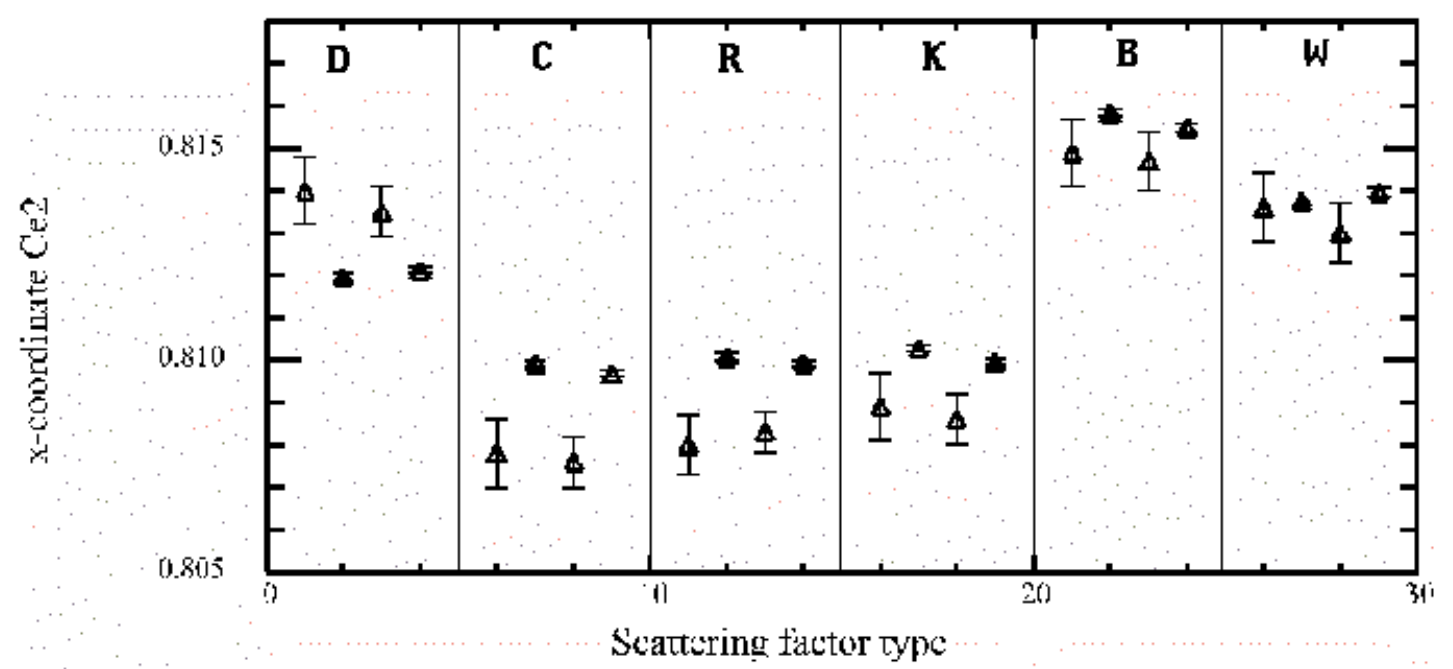

Figure 1 Refinement results for the $X$-coordinate of Ce2. Each group of 4 points represen ts one of the scattering factor tables: from left to right Doyle, Cromer, Rez, Kirkland, Bird, Weickenmeier. Within each group of 4 points the first 2 are obtained using $R_{1}$ and the last 2 using $R_{2}$. Point 1 and 3 in each group are obtained using unit weights, the other 2 using $1 / \sigma^{2}$ weights. 